\title{
Common scaling behavior in finance and macroeconomics
}

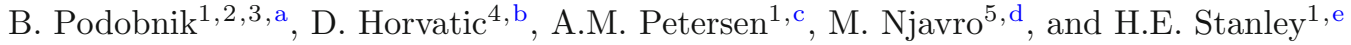 \\ 1 Center for Polymer Studies and Department of Physics, Boston University, Boston, MA 02215, USA \\ 2 Faculty of Civil Engineering, University of Rijeka, 51000 Rijeka, Croatia \\ 3 Zagreb School of Economics and Management, 10000 Zagreb, Croatia \\ 4 Department of Physics, University of Zagreb, 10000 Zagreb, Croatia \\ 5 Assia Research Center, University of St. Gallen, 9000 St. Gallen, Switzerland
}

Received 15 July 2009 / Received in final form 21 September 2009

Published online 7 November 2009 - (c) EDP Sciences, Società Italiana di Fisica, Springer-Verlag 2009

\begin{abstract}
In order to test whether scaling exists in finance at the world level, we test whether the average growth rates and volatility of market capitalization $(M C)$ depend on the level of $M C$. We analyze the $M C$ for 54 worldwide stock indices and 48 worldwide bond indices. We find that (i) the average growth rate $\langle r\rangle$ of the $M C$ and (ii) the standard deviation $\sigma(r)$ of growth rates $r$ decrease both with $M C$ as power laws, with exponents $\alpha_{w}=0.28 \pm 0.09$ and $\beta_{w}=0.12 \pm 0.04$. We define a stochastic process in order to model the scaling results we find for worldwide stock and bond indices. We establish a power-law relationship between the $M C$ of a country's financial market and the gross domestic product (GDP) of the same country.
\end{abstract}

PACS. 89.65.Gh Economics; econophysics, financial markets, business and management - 89.65.-s Social and economic systems - 89.75.Da Systems obeying scaling laws - 02.50.Ey Stochastic processes

Many real-world systems exhibit self-similarity, where fluctuations exhibit similar statistical properties at all scales $[1-3]$. As a result, these systems can be classified by the scale-free functions that characterize the underlying dynamics. In recent decades, methods from statistical physics have been extended into finance and macroeconomics, where for example, references [4,5] analyzed the fluctuations in the gross domestic product (GDP) of 152 countries and found that (i) the broad central region of the distribution of annual growth rates decays according to a Laplace distribution; and (ii) the standard deviation scales as a power law of GDP with a scaling exponent $\beta \approx 0.15$. These scaling results were also obtained for several other macroeconomic variables [5], as well as for microeconomic variables, such as the number of employees and the sales of companies [6]. The same scaling properties (i) and (ii) have found by analyzing growth dynamics of university research [7], and recently by analyzing annual growth rates for many companies comprising different financial indices [8]. In finance, the probability density function (pdf) of logarithmic returns of stock price exhibits a power-law decay in the far tails described by a inverse quartic law $[9,10]$. Recent contributions to the field indi-

\footnotetext{
a e-mail: bp@phy.hr

b e-mail: davorh@phy.hr

c e-mail: amp17@buphy.bu.edu

d e-mail: njavro@gmail.com

e e-mail: hes@bu.edu
}

cate that the far tails of the pdf of growth rates, of both microeconomic and macroeconomic variables, exhibit approximately inverse cubic power laws $[5,11]$.

In order to further investigate the scaling properties in finance and macroeconomics, first we analyze Bloomberg data on the stocks comprising a common stock index, the Nasdaq Composite Index [12]. For each stock we know the stock price and market capitalization $(M C)$ at the end of each year, where $M C$ is calculated as the number of stocks outstanding multiplied by the price of the stock. We define an annual logarithmic growth rate

$$
R_{t} \equiv \ln \left(\frac{S_{t}}{S_{t-1}}\right)
$$

where $S_{t}$ and $S_{t-1}$ are the stock prices in two consecutive years. We also analyze a data set comprising the $M C$ for 54 worldwide stock indices and 48 worldwide bond indices (from World Federation of Exchanges [13]) covering the last 16-year time horizon. In addition, we investigate the relationship between country GDP and country $M C$ (measured by the market capitalization of the national exchange where data are available).

Previous studies found that the pdf of returns exhibits a Lévy distribution in the central part and an inverse quartic power law in the tail part. For large $\Delta t$, the pdf approaches a Gaussian distribution, in agreement with the central limit theorem $[9,14-16]$. Pdfs of annual growth rate for individual companies cannot be analyzed due to data insufficiency, so we are forced to pool data of different 


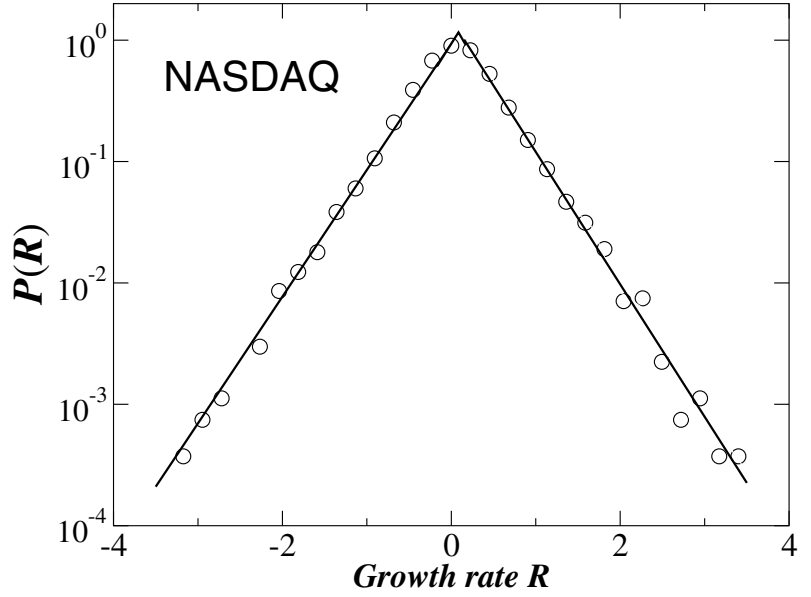

Fig. 1. Probability density functions $P(R)$ of the annual growth rates $R \equiv \ln \left(S_{t} / S_{t-1}\right)$ of stock prices $S_{t}$. Stocks comprising the Nasdaq Composite Index. For the 6-year time horizon from $1 / 1 / 2002$ to $1 / 1 / 2008$, there are $11836 R_{t}$ values. The distribution exhibits a tent-shape, implying that $P(R)$ is a Laplace function of $R$.

companies [17]. Recently, reference [8] analyzed the annual logarithmic growth rate $R_{t}$ of stocks comprising the NYSE Composite index and the Nasdaq Composite index. All the pairs $(R, M C)$ were subdivided into three groups of equal size in $M C$, and for each index and subgroup it was found that

(i) the pdf $P(R)$ follows a Laplace pdf

$$
P(R)=\frac{1}{\sqrt{2} \sigma(R)} e^{-(\sqrt{2}|R-\langle R\rangle| / \sigma(R))} .
$$

(ii) $P(R)$ is characterized by the standard deviation $\sigma=$ $\sigma(\mathrm{R})$, which depends on $M C$, where smaller $\sigma(\mathrm{R})$ corresponds to larger $M C$ values.

Here, for the 6679 stocks comprising the Nasdaq Composite Index covering the 6 -year time horizon, January 1 , 2002 to January 1, 2008, we aggregate all 11836 values of $R_{t}$ (regardless of $M C$ ) into one common data set. In Figure 1 we see that the broad central region of $P(R)$ displays a Laplace pdf - the same as we found for the three subgroups in reference [8]. Furthermore, by analyzing different US financial indices, we found that the standard deviation of the annual growth rates scales as a power law of $M C$. Does the same behavior exist for worldwide financial indices?

Global securities markets have expanded over the last decade, so we next show that the size-dependence of the previous scaling analysis, found on the US market, also holds for worldwide securities markets (larger scales). The $M C$ data are generally not publicly available for worldwide stocks, but they are for the market in total. According to the $M C$ definition, scaling of the $M C$ reflects roughly the scaling of the average stock value within a given market. We speculate that the $M C$ may serve as a good indicator for how securities markets grow at the world level. Two interesting questions are: (i) how does
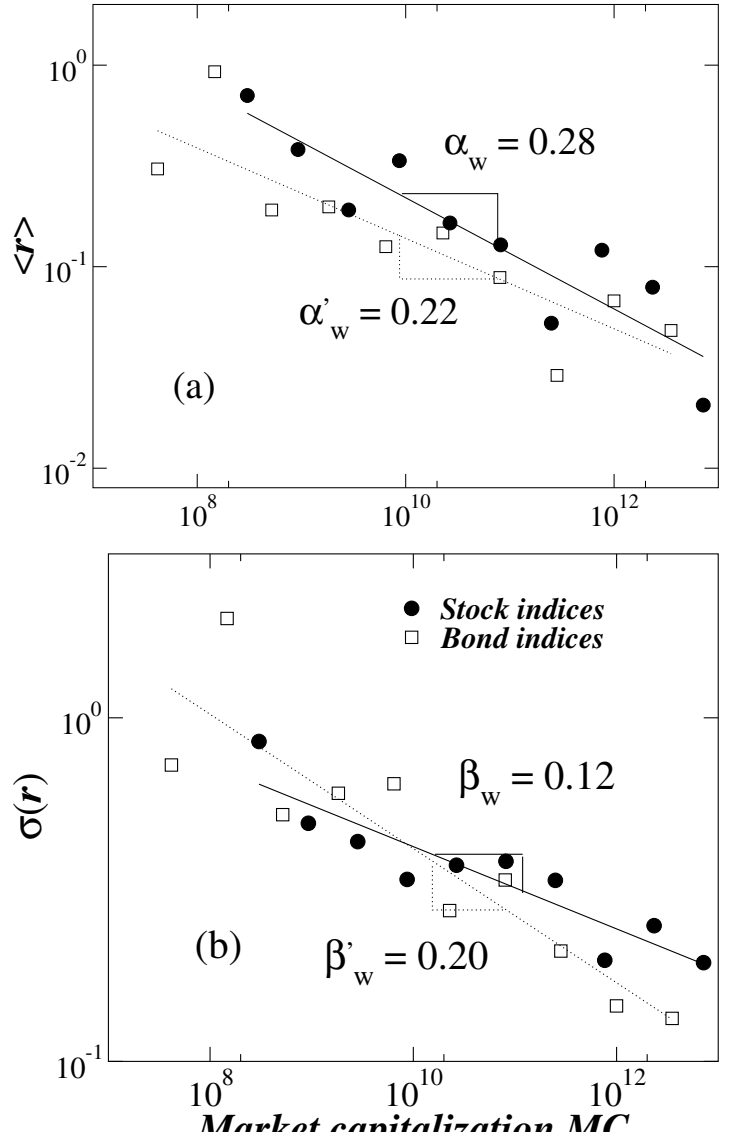

Market capitalization MC

Fig. 2. Analysis of 54 worldwide stock indices and 48 worldwide bond indices from January 1, 1991 to January 1, 2007. (a) Average annual growth rates $r_{t}$ versus $M C$ for both stock indices and bond indices. Large markets, characterized by large values of $M C$, grew slower, but are also less volatile. (b) Standard deviation $\sigma(r)$ of the annual growth rates $r_{t} \equiv$ $\ln \left(M C_{t} / M C_{t-1}\right)$ of stock indices market capitalization $\left(M C_{t}\right)$ as a function of the market capitalizations. Shown also is $\sigma(r)$ as a function of the bond market capitalization $M C$.

the $M C$ grow for different securities markets? (ii) How does the risk change for different markets?

To answer these two questions, we analyze the $M C$ for 54 different worldwide stock indices over a 16 -year period [13]. In Figure 2a we find that the average annual logarithmic growth rate $\langle r\rangle$ decreases with $M C$ as a power law

$$
\langle r\rangle \sim(M C)^{-\alpha_{w}}
$$

with exponent

$$
\alpha_{w}=0.28 \pm 0.09 .
$$

In Figure $2 \mathrm{~b}$ we show that the standard deviation $\sigma(r)$ of annual $M C$ growth rates

$$
r_{t} \equiv \ln \left(\frac{\mathrm{MC}_{t}}{\mathrm{MC}_{t-1}}\right)
$$

decreases with increasing $M C$, over more than four orders of magnitude, as a power law

$$
\sigma(r) \sim(M C)^{-\beta_{w}}
$$


with exponent

$$
\beta_{w}=0.12 \pm 0.04 \text {. }
$$

Thus, we find that larger markets, characterized by larger values of $M C$, grow slower than smaller markets, but with smaller volatility, where $\langle r\rangle$ decreases faster than $\sigma(r)$ with $M C\left(\alpha_{w}>\beta_{w}\right)$.

We also analyze 48 worldwide bond indices over a 16-year period. In Figure $2 \mathrm{a}$ we find that the standard deviation $\sigma(r)$ of annual growth rates $r_{t}$ decreases with $M C$ as a power law

$$
\sigma(r) \propto(M C)^{-\beta_{w}^{\prime}},
$$

with exponent

$$
\beta_{w}^{\prime}=0.22 \pm 0.04
$$

which appears to be different than the exponent $\beta_{w}$ obtained for world stock indices, equation (7). Figure $2 \mathrm{~b}$ shows that the average growth rate $\langle r\rangle$ decreases with $M C$ as a power law

$$
\langle r\rangle \sim(M C)^{-\alpha_{w}^{\prime}}
$$

with exponent

$$
\alpha_{w}^{\prime}=0.20 \pm 0.08
$$

Thus, moving from small to large bond markets, both $\langle r\rangle$ and $\sigma(r)$ are declining with $M C$ according to approximately the same power law $\left(\alpha_{w}^{\prime} \approx \beta_{w}^{\prime}\right)$, a result of potential interest for investors.

In order to model the observed power-law scaling behavior found in Figure 2, we introduce a discrete-time stochastic process

$$
R_{t} \equiv \ln \left(\frac{M C_{t}}{M C_{t-1}}\right)=\left(M C_{t-1}\right)^{\delta_{1}} \mu_{0} \Delta t+\left(M C_{t-1}\right)^{\delta_{2}} \sigma_{0} \eta_{t} \Delta t,
$$

motivated by the stochastic process of proportional growth rates proposed by Cox and Ross [18]. Here, $\eta_{t}$ denote i.i.d. stochastic process with Gaussian distribution with zero mean and unit variance, $\Delta t$ is time scale, $\mu_{0}$ and $\sigma_{0}$ are two free parameters, while $\delta_{1}$ and $\delta_{2}$ are two scaling exponents. Note that equation (12) reduces to geometric Brownian motion in the limit $\delta_{1}=\delta_{2}=0$. In that limit $\mu$ and $\sigma$ represent the mean and the standard deviation, respectively. However, if $\delta_{1} \neq 0$ and $\delta_{2} \neq 0$, according to equation (12) both mean and standard deviation change over time, and these are the regularities we find in the $M C$-dependence of $\langle R\rangle$ (Fig. 2a) and $\sigma(R)$ (Fig. 2b), respectively. In Figures $3 \mathrm{a}-3 \mathrm{~b}$ an arbitrarily time series generated by the stochastic process of equation (12) shows $M C$-dependence of $R$ and $\sigma(R)$ in agreement with what we found in empirical data. Since $M C_{t}$, stock price $S_{t}$, and number of stocks outstanding $N$ are related as $M C_{t}=N S_{t}$, if we assume that $N$ does not change, from equation (12) we easily obtain a similar stochastic process for stock-price dynamics

$$
R_{t} \equiv \ln \left(\frac{S_{t}}{S_{t-1}}\right)=\left(S_{t-1}\right)^{\delta_{1}} \mu \Delta t+\left(S_{t-1}\right)^{\delta_{2}} \sigma \eta_{t} \Delta t
$$

where $\mu=N^{\delta_{1}} \mu_{0}$ and $\sigma=N^{\delta_{2}} \sigma_{0}$, where $\mu_{0}$ and $\sigma_{0}$ are defined in equation (12).
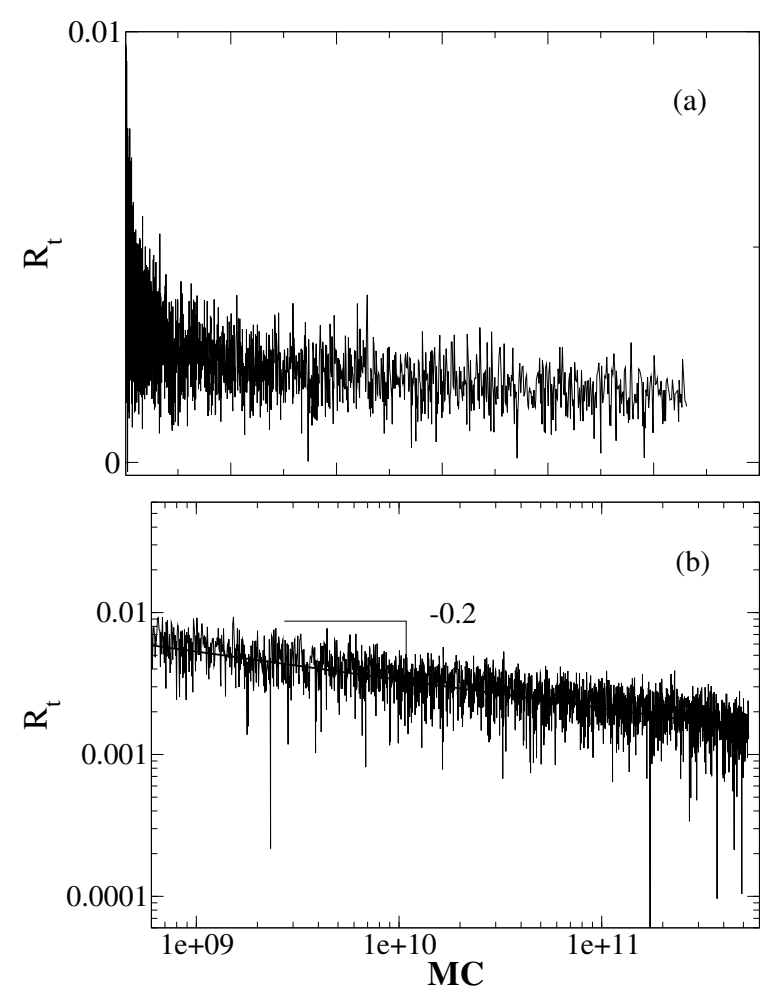

Fig. 3. Time series shows $M C$-dependence of the growth rate $R$ of process of equation (12) in agreement with empirically found $M C$-dependence of $\langle R\rangle$ (Fig. 2a) and $\sigma(R)$ (Fig. 2b). (b) is a log-log plot of (a). In (a) we see that the fluctuations are larger for smaller values of $M C$. The time series we obtain with $\delta_{1}=\delta_{2}=0.2, \sigma=0.1$ and $\mu=0.35$ and initially chosen $M C=10^{5}$. After each step we calculate a new $M C$, as $M C_{t}=$ $M C_{t-1} \exp \left(R_{t}\right)$.

Next, we investigate if the stochastic process proposed in equation (12) can generate time series for which the pdf $P(R)$ follows a Laplace pdf, as found in empirical data in Figure 1. By using the discrete-time process of equation (12), we choose for example $\gamma=-0.15[6], \mu=0.07$, $\sigma=2$ and generate $N=100000$ time series $M C_{t}$ all of the same length $n=20$. For each time series we choose initial $M C_{0}$ from the homogeneous distribution. Finally we pool $N n$ values of $R_{t}$, and calculate the pdf $P(R)$. In Figure 4 we find that the central part of $P(R)$ is consistent, over more than six orders of magnitude, with a Laplace distribution, and so the process is capable to reproduce the empirical findings in Figure 1.

It is known that during the periods of economic growth, the financial sector measured by $M C$ generally grows faster than the real economy, measured by GDP. On the contrary, the $M C$ declines substantially faster than the GDP during periods of economic recession. However, one expects that, in the long run, financial and macroeconomic indicators should follow each other to a certain degree. In order to investigate a possible relationship between finance and macroeconomics, it was found that the periods with high volatility are commonly associated with important events in each country more often than with global events. The October 1987 crash is the only global 


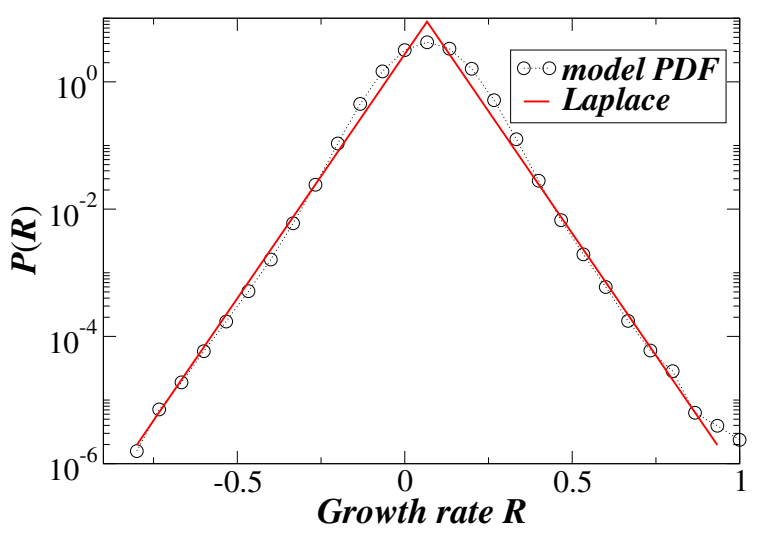

Fig. 4. (Color online) Linear-log plot of pdf $P(R)$ of growth rate $R$ generated by the process of equation (12). We set $\gamma=-0.15, \mu_{0}=0.07$, and $\sigma=2$ and generate 100000 time series each of 20 values of $R_{t}$. The initial $M C$ for each time series we take from the homogeneous distribution - first we generate a random number from zero to $10^{9}$, and to that random number then we add $10^{6}$. For the process of equation (12) $P(R)$ in linear-log plot exhibits double-exponential functional form (Laplace) in the broad central region.

event that significantly increased volatility in many worldwide markets [19-21].

Since macroeconomics and finance exhibit similar scaling behavior, we expect that GDP and $M C$ should have a strong relationship. Hence, we analyze whether $M C$ and GDP scale at the world level. For two particular years, 1990 and 2006, we show in Figure 5 that $M C$ approximately follows a power law in GDP,

$$
\mathrm{MC} \propto(\mathrm{GDP})^{\gamma}
$$

For all 17 years between 1990 and 2006 we calculate the power-law exponent and find $\langle\gamma\rangle=1.1 \pm 0.10$.

We thank J. Cvitanic, X. Gabaix, and M.A. Salinger for valuable discussions and the Ministry of Science of Croatia and the NSF for financial support.

\section{References}

1. R.N. Mantegna, H.E. Stanley, An Introduction to Econophysic (Cambridge U. Press, Cambridge, 2000)

2. Z. Eisler, I. Bartos, J. Kertesz, Adv. Phys. 57, 89 (2008)

3. Z. Eisler, J. Kertesz, Phys. Rev. E 73, 046109 (2006)

4. Y. Lee et al., Phys. Rev. Lett. 81, 3275 (1998)

5. B. Podobnik et al., Phys. Rev. E 77, 056102 (2008)

6. M.H.R. Stanley et al., Nature 379, 804 (1996); L.A.N. Amaral et al., J. Phys. France 7, 621 (1997)

7. V. Plerou et al., Nature 400, 433 (1999)

8. B. Podobnik, D. Horvatic, A.M. Petersen, H.E. Stanley, Europhys. Lett. 85, 50003 (2009)

9. P. Gopikrishnan et al., Phys. Rev. E 60, 5305 (1999); V. Plerou et al., Phys. Rev. E 60, 6519 (1999)
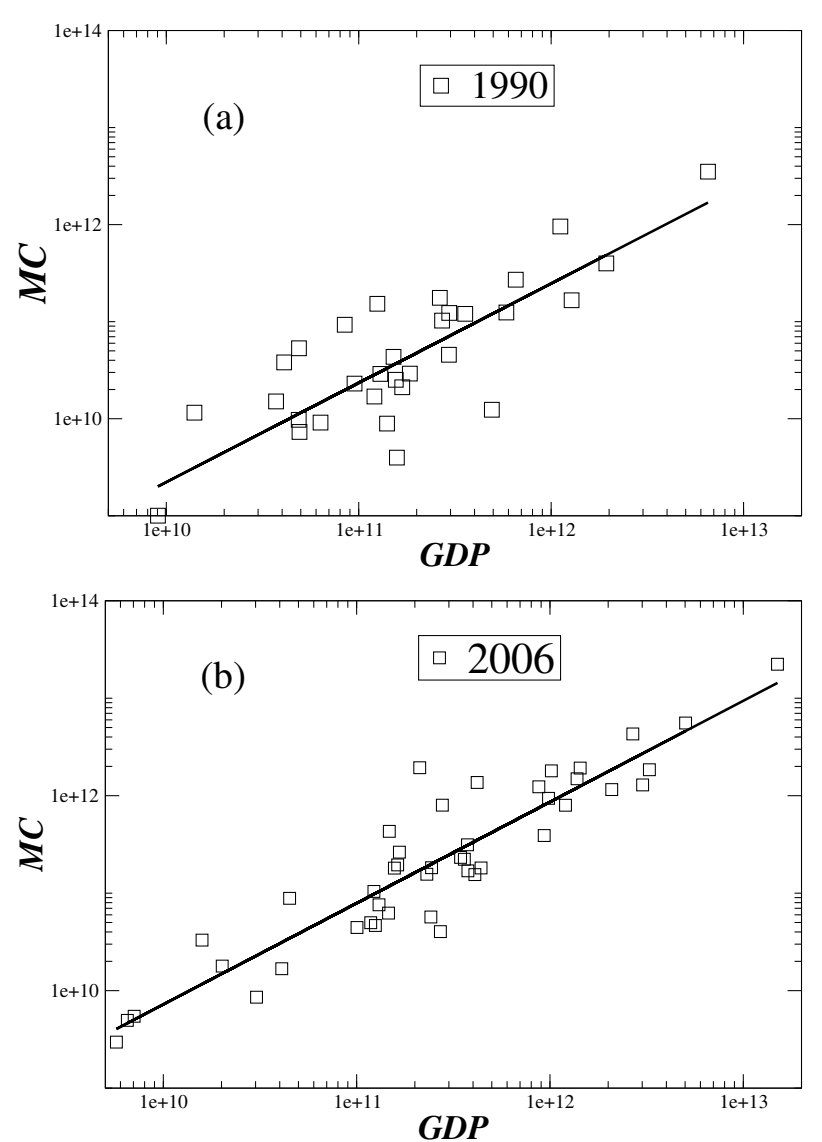

Fig. 5. Market capitalization $(M C)$ versus gross domestic product (GDP) exhibits a power law across countries. We analyzed each year from 1990-2006, and here we show the powerlaw dependence for (a) 1990 and (b) 2006.

10. T. Lux, Appl. Fin. Econ. 6, 463 (1996)

11. D. Fu et al., Proc. Natl. Acad. Sci. USA 102, 18801 (2005); M. Riccaboni et al., Proc. Natl. Acad. Sci. USA 105, 19595 (2008)

12. The data are available at Bloomberg http://www. bloomberg. com

13. Publicly available at http://www . world-exchanges .org/WFE/home . asp? menu $=195$

14. Empirical Science of Financial Fluctuations: The Advent of Econophysics, edited by H. Takayasu (Springer, Berlin, 2002)

15. R.N. Mantegna, H.E. Stanley, Nature 376, 46 (1995)

16. B. Podobnik, P.Ch. Ivanov, Y. Lee, H.E. Stanley, Europhys. Lett. 52, 491 (2000)

17. E.F. Fama, K.R. French, J. Finance 47, 427 (1992)

18. J.C. Cox, S.A. Ross, J. Financ. Econ. 3, 145 (1976)

19. W. Bailey, Y.P. Chung, J. Financ. Quant. Anal. 30, 541 (1995)

20. G. Bekaert, C.R. Harvey, J. Financ. Econ. 43, 29 (1997)

21. R. Aggarwal, C. Inclan, R. Leal, J. Financ. Quant. Anal. 34, 33 (1999) 